\title{
Impact of Transportation Problem's Unbalance on Difference between Initial and Optimal Solution
}

\author{
Mario GALIĆ, Zlata DOLAČEK-ALDUK, Uroš KLANŠEK
}

\begin{abstract}
This paper deals with the impact of transportation problem's (TP) unbalance on difference between the initial and optimal solution. An analysis based on the executed numerical experiments roughly reveals how the unbalance of linear TP affects differences between objective function values of initial feasible solutions generated by most commonly applied approaches (such as least cost method and Vogel's approximation method) and optimal solutions. Furthermore, this research addresses the question whether the form and/or the number of decision variables has an influence on such differences. The obtained results have indicated scattered, but roughly visible linear relation between unbalance of linear TP and difference between objective function values of initial and optimal solutions.
\end{abstract}

Keywords: initial solution; optimal solution; unbalanced transportation problem

\section{INTRODUCTION}

Transportation problem (TP), ever since it was introduced [1], is a relevant scientific and professional topic. Although the nature of TPs is relatively simple, the problem itself is quite often found in industry and many times identified as a challenging task. In its most common form, TP is defined as a linear optimization problem of simultaneous transportation of some good from multiple sources to multiple destinations in such order that the total transportation costs, which are linearly dependent on the quantities of transported good, should be as minimal as possible. As well, in its basic formulation, the total supply is equal to the total demand, in literature known as the balanced TP (BTP). However, the aforesaid equality often does not hold in real-life situations. At this point, the unbalanced TPs (UBTP) are found in cases where: i) the supply of good is larger than demand, which is considered as a simpler type of UBTP and solvable by modifying the original problem into a $\mathrm{BTP}$; or ii) where the demand exceeds the supply.

In dealing with both types of TPs, researchers were mainly focused on mathematical improvements of solving methods for gaining initial solutions and comparing them with optimal ones [2-6]. Furthermore, some authors addressed the methods for solving specific TP's with uncertainties in input parameters [7-12] and optimization methods for handling TP's with nonlinearities [13-18]. However, previous studies have overlooked the questions: does the magnitude of problem's unbalance affect the difference between the initial feasible solution and the optimum one, or does the problem's matrix form influence it as well. Those two questions are main motivations for the research presented in the following text.

This article analyses how the magnitude of TP's unbalance, in case when total demand is higher than total supply, affects the initial feasible solution generated by basic approaches (least cost method, LCM; Vogel's approximation method, VAM) in comparison with optimal solution gained by optimization method (such as Simplex).

In this way, the main aim of the paper is to share some new information for practitioners, who often rely their decision about transportation on feasible solutions, as well as for research community that is involved in development of algorithms for solving UBTPs concerning their structural unbalances and matrix forms.

\section{UBTP FORMULATION}

Linear UBTP, in which the total demand exceeds the total supply, contains the objective function Eq. (1), which is the same as in classical linear TPs:

$\min Z=\sum_{i=1}^{m} \sum_{j=1}^{n} c_{i j} \cdot x_{i j}$

where $Z$ denotes the total transportation costs while $c_{i, j}$ and $x_{i, j}$ represent cost parameters and transporting flows from suppliers $i$ to demanders $j$ contained in the criterion of optimization.

The presented cost objective function is subjected to structural constraints, which actually make the difference between classical TP and UBTP. The total demand $b_{j}$ is set here as strictly higher than the total supply $a_{i}$, which means that the total quantity of good needs to match the total demand but it is less than the total supply, see Eqs. (2) and (3).

$\sum_{i=1}^{m} a_{i}<\sum_{j=1}^{n} b_{j}$
$\sum_{j=1}^{n} x_{i j}=a_{i} ; \quad \sum_{i=1}^{m} x_{i j}<b_{j} ; \quad x_{i j} \geq 0$

From the constraints given in Eq. (2), it is obvious that the ratio of total demand and total supply is strictly greater than one. This ratio is denoted here as the unbalance ratio $U B R$ and formulated by Eq. (4).

$U B R=\frac{\sum_{j=1}^{n} b_{j}}{\sum_{i=1}^{m} a_{i}}>1,0$

The influence of $U B R$ increment on the efficiency of initial feasible solutions generated by LCM and VAM is 
analysed here by comparing objective function values of initial $\left(Z^{0}\right)$ and optimal solutions $\left(Z^{*}\right)$ gained by Simplex, see Eq. (5).

$$
f(U B R)=\frac{Z^{0}}{Z^{*}}
$$

From the mathematical point of view, UBR can be any positive real number. However in practical sense, the difference between the total demand and total supply has to be analysed from the viewpoint of necessity for simultaneity of transport, as in $[12,19]$, or priorities among demands. Therefore, this paper considers only those situations where $U B R$ at most equals two, i.e. those cases where the total demand is maximally two times higher than the total supply. In this way, the interval of mentioned unbalance is taken as $U B R \in[1,2]$.

\section{NUMERICAL ANALYSIS OF UBR IMPACT ON INITIAL SOLUTIONS}

\subsection{Methodology of the Experiment}

The experiment started by setting the lower and upper bounds of intervals from which the input parameters were generated, coressponding to those in paper [12]. Input parameters comprised unit transportation costs $c_{i j}$ (generated from the interval of integer values from 10 to 75), as well as supply and demand quantities, i.e. $a_{i}$ (generated from the interval of integer values from 50 to 95) and $b_{j}$ (generated from the interval of integer values from 50 to 95 ) respectively.

In the second step, TP models were generated in the form of $5 \times 5,5 \times 10$ and $10 \times 10$ matrices, starting with BTP (i.e. at $U B R=1$ as the lower bound) after which $U B R$ was increased by increment of 0,05 until it reaches the value of 2 . For each $U B R$ value, 20 models were generated in order to get average differences between initial and optimal solutions meaning that linear models, describing relationships between model's ubalance ratio and difference between initial and optimal solution, are based on separate 400 generated UBTP cases in three matrix forms. Models and algorithms were structured in Microsoft Excel VBA and connected to Matlab.

The final step was the extractions of results and curve fitting, which were handled graphically and numerically, as well as the analysis of statistical data, what was done in Matlab.

The most common process of solving any type of TP starts with model formulation. Further step is to apply a suitable method for generating the initial feasible solution (e.g. NWCM, LCM, VAM, etc.). The initial solution represents a feasible program for $\mathrm{TP}$, which serves as a starting point for iterative procedure of its improvement in order to obtain the optimal solution by selected method (e.g. Simplex).

It is necessary to underline that it is possible that the techniques for generating initial solutions can also find optimal solutions, but it is still necessary to undertake the optimality tests on them by optimization methods. At this point, VAM represents an approach that may frequently generate such starting points for solving TPs that also appear to be their optimal solutions and therefore it is noted to be the most developing method among the existing ones. Initial solutions generated by north-west corner method (NWCM), unlike those found by LCM and VAM, highly depend on the distribution of $c_{i j}$ values over the matrix meaning that if $c_{i j}$ values on the matrix diagonal, connecting nort-west and south-east cell of the matrix, are significantly greater than those which are above and under the diagonal, the variations of the differences between initial and optimal solutions can be significant. Therefore, the analysis in this paper was focused mainly on LCM and VAM results, while NWCM outputs are presented as accompanying data valid for cases with uniformly distributed $c_{i j}$ values in TP model's matrix, because in this research the $c_{i j}$ values in the matrix were randomly generated equally for all cases.

The process flow usually applied for solving UBTPs is shown in Fig. 1.

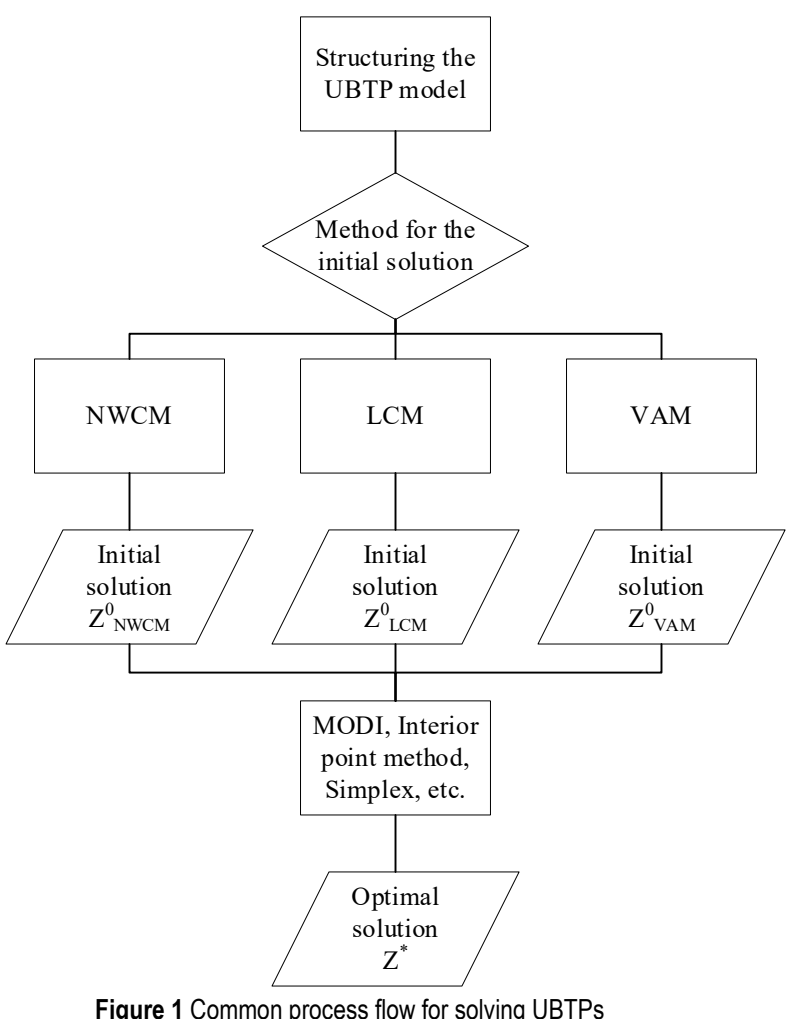

\subsection{Results}

The first set of generated cases of UBTP was set in quadratic matrix form where five potential sources were supplying five given destinations. The results have roughly demonstrated an almost linear correlation between initial solutions generated by LCM and VAM, and optimal solutions, see Tab. 1. Note here that results for NWCM were not taken into comparison but only serve as accompanying data. However, in case of VAM, dependence $f(U B R)$ is scattered at coefficient of determination $R^{2}=0,4453$ although moderate uphill relation between initial feasible results and optimal solutions can be found. In case of LCM, coefficient of determination $R^{2}=0,8865$ indicates to a stronger uphill linear relation between objective function values of initial solutions and those for the optimal ones. 
Table 1 Results for $5 \times 5$ problems

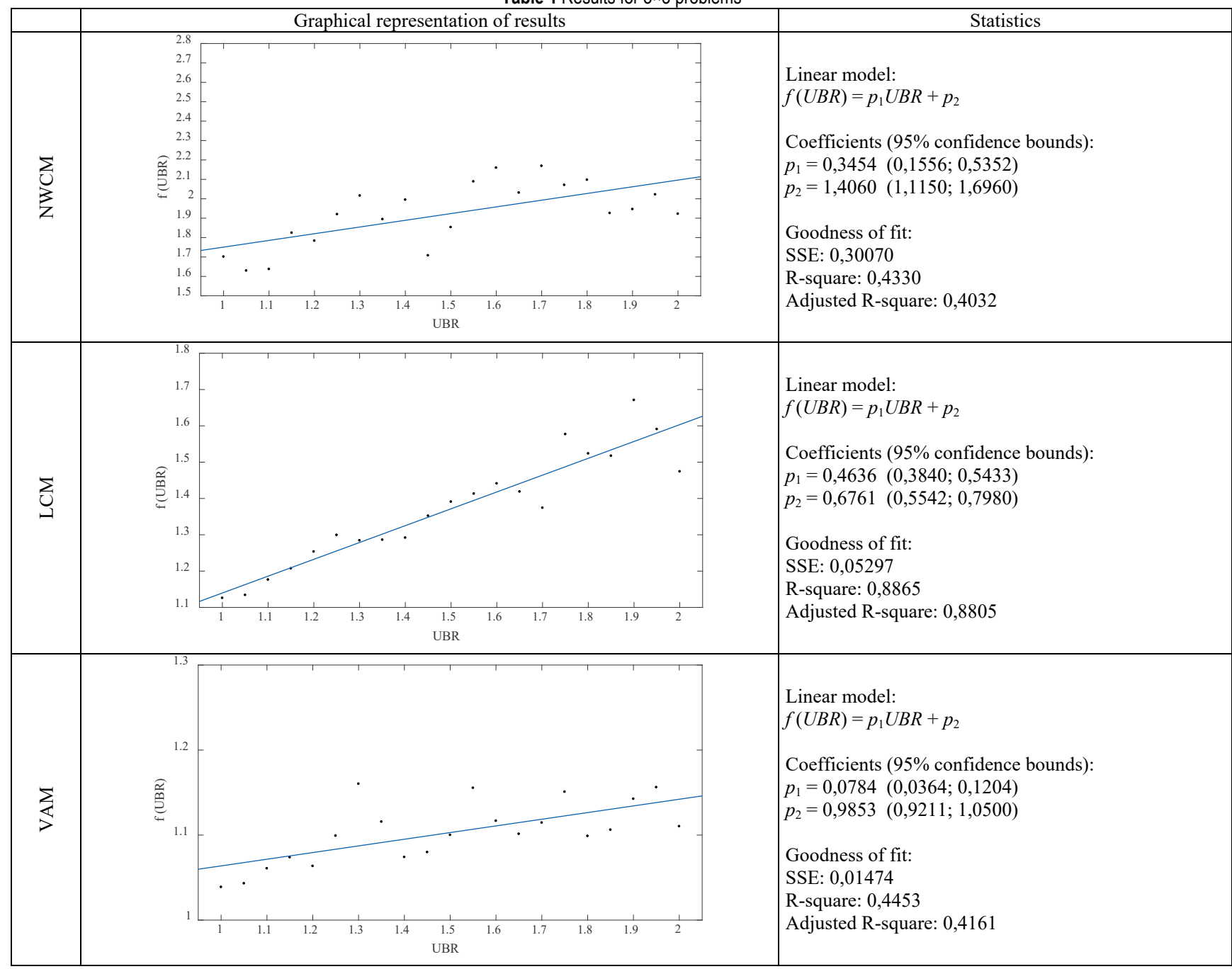

Table 2 Results for $5 \times 10$ problems

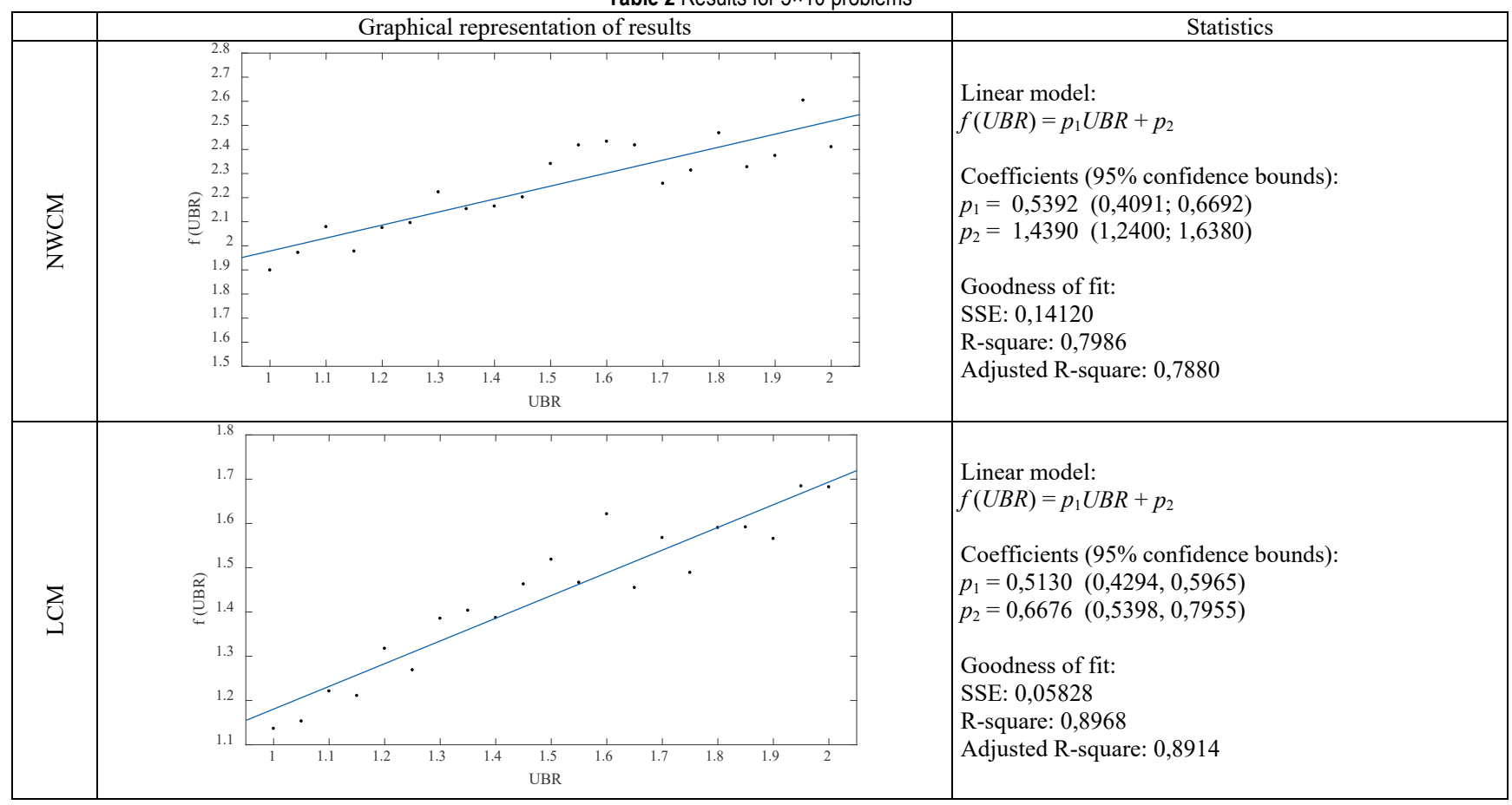


Table 2 Results for $5 \times 10$ problems (continuation)

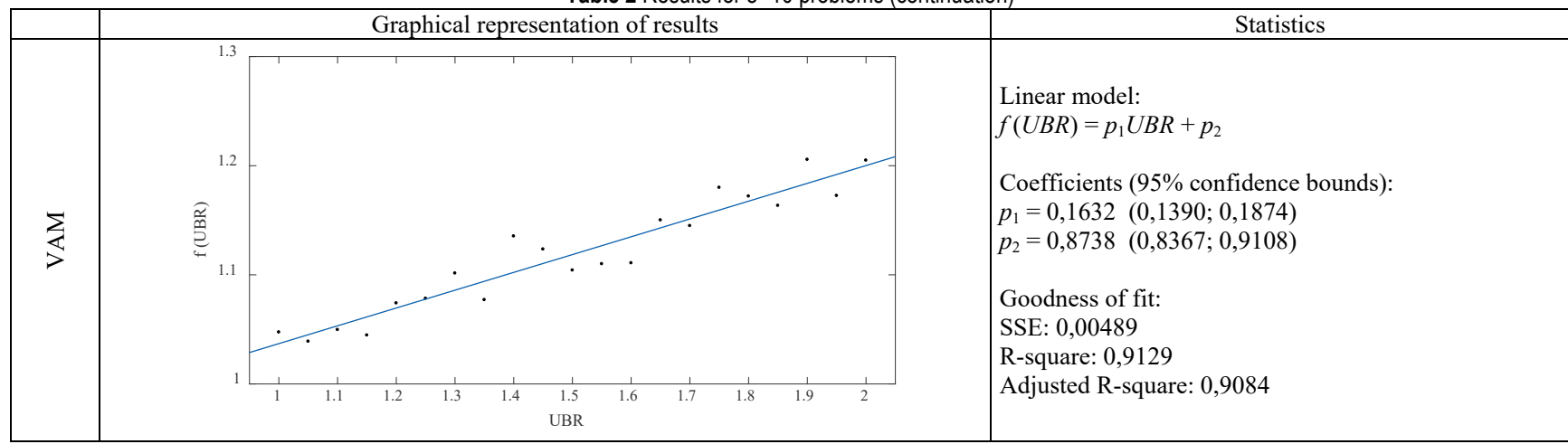

Table 3 Results for $10 \times 10$ problems

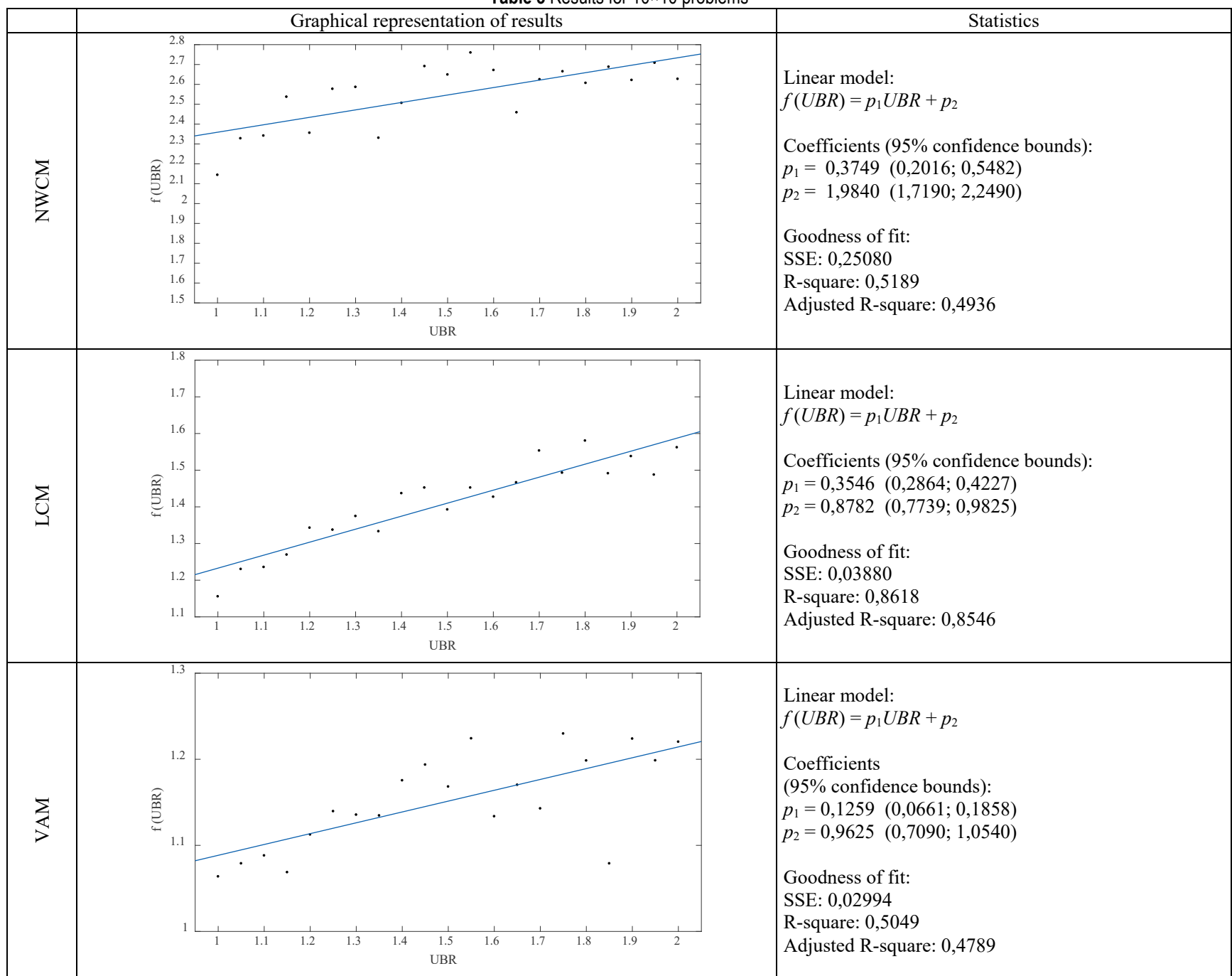

In the second set of generated cases, the UBTP matrix was changed to a form of $5 \times 10$ (i.e. five potential sources are supplying 10 destinations) for the aim of investigating how the matrix form and slight increase in number of decision variables, as well as the problems' unbalance, affect the differences between objective function values of initial solutions and the optimal ones. The results have shown (Tab. 2) that the non-quadratic form of the problem gives a rough, but clearer linear relationship. Applied methods provided average initial solutions in a reasonably stronger positive linear relationship in regard to the optimal solution. Coefficients of determination were higher, i.e. $R^{2}$ $=0,8968$ in case of LCM and $R^{2}=0,9129$ for VAM.
At this point, it was not completely clear whether the form and/or the increase in number of decision variables have strengthened the linear relationship. Therefore, the third set of UBTPs was generated, solved and analysed.

The final set of cases included UBTPs which were defined applying quadratic matrices with increased number of variables, i.e. in form of $10 \times 10$ problems. This set was used to investigate whether the increase in number of decision variables, the matrix form or both, has an impact on the difference between initial and optimal solution. The obtained results have demonstrated that the established relationship is similar to the one found in the first set of generated UBTPs (i.e. for $5 \times 5$ problems), see Tab. 3 . The 
achieved results for VAM were scattered and coefficients of determination roughly indicated a moderate positive linear relationship between initial and optimal solutions, while slightly stronger uphill linear correlation was identified for LCM.

\section{DISCUSSION OF THE RESULTS}

Although numerical experiments were done on 400 small-scale UBTPs in three matrix forms, it is difficult to completely generalize findings from received outputs but some conclusions can however be highlighted. In this context, the obtained results have indicated clear, although in some cases rough, linear relation between unbalance of linear TP and difference between objective function values of initial and optimal solutions. In addition, the problem matrix form exposed a bit clearer linear relation.

Due to NWCM's sensitivity in terms of distribution of $c_{i j}$ values in the UBTP matrix, it is hard to make general conclusions regarding the impact of its unbalance or form of the matrix on the difference between initial and optimal solutions gained by this method. However, in cases of uniformly distributed matrix of $c_{i j}$ values the dependence of the problem's unbalance is clearer in non-quadratic matrix form.

In cases of the LCM, results have shown a strong linear dependence of the problem's unbalance, with the highest determination coefficient and scatter in the $95 \%$ confidence bounds, regardless of the matrix form of the problem.

The clearest dependence of the matrix form of the problem on the difference between the initial and optimal results was noted in case of VAM. While in cases of quadratic forms the relationship was noted as moderate linear positive with scattered results in the $95 \%$ confidence bounds, in cases of non-quadratic matrix form of the problem the coefficient of determination was noted as the highest in the experiment with clear positive linear trend of the relationship between initial and optimal solutions.

\section{CONCLUSIONS}

TPs are rarely perfectly balanced in real-time circumstances. Their unbalance or asymmetric demand relative to supply and vice versa is a common fact. This article was intended to find out how the unbalance of TP affects differences between objective function values of initial feasible solutions generated by most commonly applied methods (i.e. LCM and VAM) and optimal solutions. As well, research also addressed the question whether the form or the number of decision variables hold an influence on such differences.

Experiments on a set of small-scale problems roughly revealed that the number of variables had no effect on differences between initial and optimal solutions as long as the problem matrix form was quadratic. For quadratic problems, VAM gave scattered but moderate positive uphill linear relationship between objective function values of initial solutions and optimal ones while such connection was more strongly pronounced in results of LCM. As regards non-quadratic problem forms, the considered methods have exposed fairly visible linear relationships.

\section{Acknowledgements}

The authors acknowledge the financial support from the Slovenian Research Agency (research core funding No. P2-0129).

\section{REFERENCES}

[1] Hitchcock, F. L. (1941). The distribution of a product from several sources to numerous localities. Journal of Mathematics and Physics, 20(1-4), 224-230. https://doi.org/10.1002/sapm194120122

[2] Shimshak, D., Kaslik J. A., \& Barclay T. (1981). A modification of Vogel's approximation method through the use of heuristics. INFOR: Information Systems and Operational Research, 19(3),259-263. https://doi.org/10.1080/03155986.1981.11731827

[3] Goyal, S. K. (1984). Improving VAM for unbalanced transportation problems. Journal of the Operational Research Society, 35(12), 1113-1114. https://doi.org/10.1057/jors.1984.217

[4] Ramakrishnan, C. S. (1988). An improvement to Goyal's modified VAM for the unbalanced transportation problem. Journal of the Operational Research Society, 39(6), 609610. https://doi.org/10.1057/jors.1988.101

[5] Balakrishnan, N. (1990). Modified Vogel's approximation method for the unbalanced transportation problem. Applied Mathematics Letters, 3(2), 9-11. https://doi.org/10.1016/0893-9659(90)90003-T

[6] Korukoğlu, S. \& Ballı, S. (2011). A Improved Vogel's Approximatio Method for the Transportation Problem. Mathematical and Computational Applications, 16(2), 370381. https://doi.org/10.3390/mca16020370

[7] Adlakha, V., Kowalski, K., \& Lev, B. (2006). Solving transportation problems with mixed constraints. International journal of management Science and Engineering management, 1(1), 47-52. https://doi.org/10.1080/17509653.2006.10670996

[8] Kumar, A. \& Kaur, A. (2012). Methods for solving unbalanced fuzzy transportation problems. Operational Research, 12(3), 287-316. https://doi.org/10.1007/s12351-010-0101-3

[9] Hussain, R. J. \& Kumar, P. S. (2012). Algorithmic approach for solving intuitionistic fuzzy transportation problem. Applied mathematical sciences, 6(80), 3981-3989. Retrieved from http://www.m-hikari.com/ams/ams-2012/ams-77-802012/index.html

[10] Che, Z. H. (2012). A particle swarm optimization algorithm for solving unbalanced supply chain planning problems. Applied Soft Computing, 12(4), 1279-1287. https://doi.org/10.1016/j.asoc.2011.12.006

[11] Galić, M., Dolaček-Alduk, Z., \& Završki I. (2013).The importance of additional criteria in solving transportation problem. Paper presented at the Creative Construction Conference 2013, Budapest Hungary. Retrieved from $\mathrm{http} / / / 2013$.creative-construction-conference.com/

[12] Galić, M., Završki, I., \& Dolaček-Alduk, Z. (2016). Methodology and algorithm for asphalt supply chain optimization. Tehnički vjesnik - Techical Gazette, 23(4), 1193-1199. https://doi.org/10.17559/TV-20150623140015

[13] Michalewicz, Z., Vignaux, G. A., \& Hobbs M. (1991). A nonstandard genetic algorithm for the nonlinear transportation problem. ORSA Journal on Computing, 3(4), 267-384. https://doi.org/10.1287/ijoc.3.4.307

[14] Jo, J.-B., Li, Y., \& Gen, M. (2007). Nonlinear fixed charge transportation problem by spanning tree-based genetic algorithm. Computers \& Industrial Engineering, 53(2), 290298. https://doi.org/10.1016/j.cie.2007.06.022 
[15] Klanšek, U. (2014). Solving the nonlinear discrete transportation problem by MINLP optimization. Transport, 29(1), 1-11. https://doi.org/10.3846/16484142.2013.815134

[16] Xie, F. \& Jia, R. (2012). Nonlinear fixed charge transportation problem by minimum cost flow-based genetic algorithm. Computers \& Industrial Engineering, 63(4), 763778. https://doi.org/10.1016/j.cie.2012.04.016

[17] Anholcer, M. (2015). The nonlinear generalized transportation problem with convex costs. Croatian Operational Research Review, 6(1), 225-239. https://doi.org/10.17535/crorr.2015.0018

[18] Lu, J., Gupte, A., \& Huang, Y. (2018). A mean-risk mixed integer nonlinear program for transportation network protection. European Journal of Operational Research, 265(1), 277-289. https://doi.org/10.1016/j.ejor.2017.07.025

[19] Chiu, Y. P., Chiang, K.-W., Chiu, S. W., \& Song, M.-S. (2016). Simultaneous determination of production and shipment decisions for a multi-product inventory system with a rework process. Advances in Production Engineering \& Management, 11(2), 141-151.

https://doi.org/10.14743/apem2016.2.216

\section{Contact information:}

Mario GALIĆ, Assistant Professor, PhD

(Corresponding author)

Josip Juraj Strossmayer University of Osijek,

Faculty of Civil Engineering Osijek,

Vladimira Preloga 3, 31000 Osijek, Croatia

mgalic@gfos.hr

Zlata DOLAČEK-ALDUK, Associate Professor, PhD

Josip Juraj Strossmayer University of Osijek,

Faculty of Civil Engineering Osijek,

Vladimira Preloga 3, 31000 Osijek, Croatia

zlatad@gfos.hr

Uroš KLANŠEK, Associate Professor, PhD

University of Maribor,

Faculty of Civil Engineering, Transportation Engineering and Architecture,

Smetanova ulica 17, 2000 Maribor, Slovenia

uros.klansek@um.si 